\title{
Restraint Strategy for Chaos in Auto Gauge Control System
}

\author{
Hui Wang ${ }^{1, *}$, Weiwei Zhang ${ }^{2}$, Xiangkui $\mathrm{Li}^{2}$ and Tianhui Liu ${ }^{2}$ \\ ${ }^{I}$ People's Public Security University of China, Beijing, China, 100038 \\ ${ }^{2}$ Tianjin Reserarch Institute of Electric Science Co. LTD. Tianjin, China, 300180
}

\begin{abstract}
In this paper, conditions of the chaos in auto gauge control system are suggested and proved on the basis of the roll eccentricity model. It is proposed that the eccentricity disturbance of roll may cause chaotic phenomena in the rolling process. By analyzing four kinds of expandable and shrinkable factors of variable universe fuzzy controller, it is suggested that the contradiction between fuzzy control rules and control accuracy can be solved effectively by HACO-VUFC method. Simulation shows that the product quality can be improved by chaos inhibition.
\end{abstract}

Keywords: Chaos, Auto Gauge Control (AGC), Duffing equation, smale-horseshoe chaos

\section{INTRODUCTION}

During rolling of strip, eccentricity compensation has been the most important factor influencing the quality of strip steel in the auto gauge control system (AGC) [1]. It is proved that AGC is one of the nonlinear complex system [2]. Because there are several signals cannot be measured directly and be used as the feedback control signal. So the AGC system has become a delay system. This system itself may generate chaos probably [3]. The trajectory of roll eccentricity motion is in compliance with the law of sine. The duffing equation is stated as roll motion equation generally [4]. Now chaos related to nonlinear systems has become a hot topic in engineering applications. Since the existence of interferences that chaos may be produced in thickness system of AGC if certain conditions are met. So the strip quality will be affected because of chaos.

Therefore, a new roll eccentric model of AGC system is established and many kinds of conditions causing the chaos are analyzed detailed in the paper. Finally methods to inhibit chaos are proposed and simulation results are shown the effectiveness about different control methods.

\section{MILL ECCENTRIC MODEL}

In the rolling process, the periodic changes between rolls caused by the irregular shape of roll and other components are named eccentricity [5], as can be seen from Fig. (1). Fig. (1a) shows that there will be no roll eccentricity when the roller circumference is homogeneous. Due to the existence of interference, the thickness difference is shown in Fig. (1c) as line 1.
However, in actual production process either the asymmetry of roller or the inadequate combination of bearings leads to the roll eccentricity, marked in Fig. (1b). From another point, this eccentricity will cause the periodic changes between the gap and the rolling thickness, the error of thickness is shown in Fig. (1c) as line 2. But generally the rolling thickness deviation may be about $10 \mu \mathrm{m}$. So for high-fidelity rolling the eccentricity cannot be ignored. In order to improve the quality of strip the eccentricity compensation must be taken.

Because of the friction and deformation the damping force exists between the contact arc of roller and rolled piece. Normally $k_{0} x$ is damping force ( $k_{0}$ means damping coefficient), and the elastic potential energy express as

$U=\frac{1}{2} k_{1} x^{2}+\frac{1}{4} k_{2} x^{4}$

Where, $k_{1}$ and $k_{2}$ are coefficients related to the elastic deformation. In comparison with $k_{1}, k_{2}$ is smaller.

The resilience about AGC is

$f=-\frac{d U}{d x}=-k_{1} x-k_{2} x^{3}$

Then the eccentricity force may be shown as

$F=\sum_{i=1} F_{i} \cos \left(\omega_{i} t+\theta\right)$

The roll motion equation may be labeled as

$-k_{0} \dot{x}-k_{1} x-k_{2} x^{3}+F \cos \omega t=m \ddot{x}$

Where, $k_{0}$ means damping coefficient, $k_{1}$ means the coefficient related to the elastic deformation, $x$ is the 


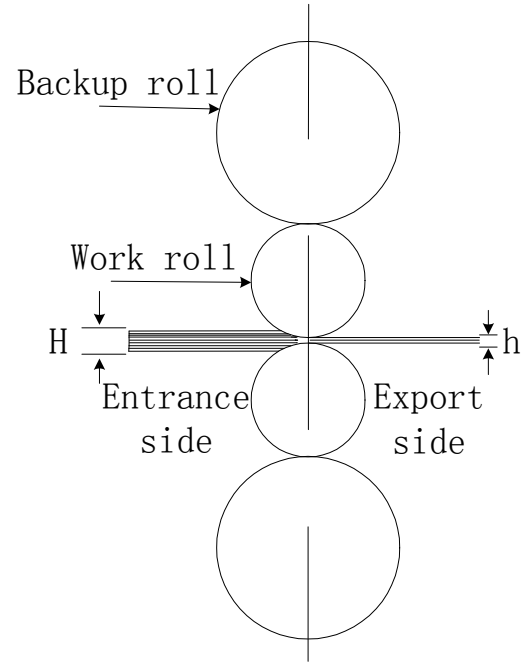

a. Non-eccentricity

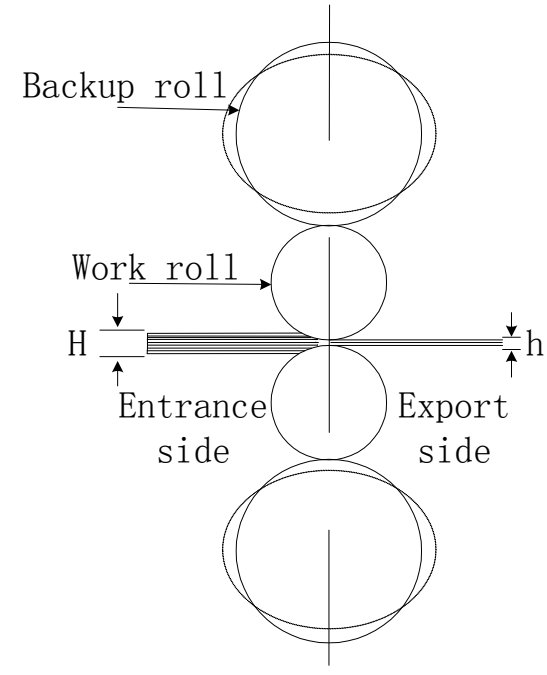

b. Eccentricity

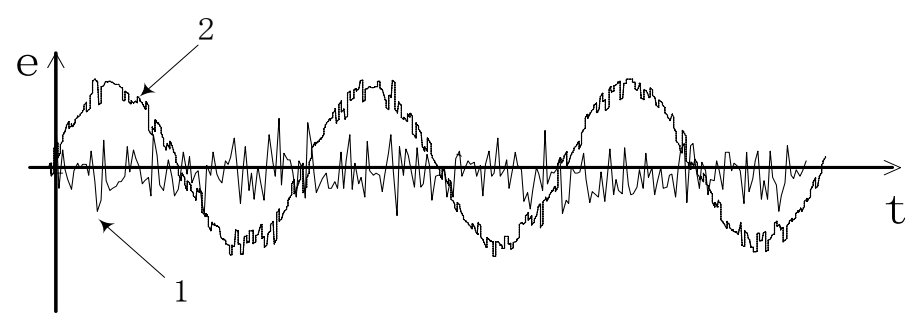

c. Thickness deviation

Fig. (1). Diagram of roll eccentricity.

eccentricity displacement of roll, $F$ and $\omega$ indicate amplitude and angular frequency of the eccentricity force separately. The equation can be transformed to the form as following.

$\ddot{x}+\frac{k_{0}}{m} \dot{x}+\frac{k_{1}}{m} x+\frac{k_{2}}{m} x^{3}-\frac{F}{m} \cos \omega t=0$

Let $k_{0} / m=\mu, \quad k_{1} / m=-\alpha, \quad k_{2} / m=-\gamma, \quad F / m=\delta$, then

$\ddot{x}+\mu \dot{x}-\alpha x-\gamma x^{3}=\delta \cos \omega t$

This equation shows that the chaotic may be appeared. In the following part, the Smale-horseshoe theory and subharmonic orbits theory is used to give the relationship between the chaotic phenomena and system parameters [6].

\section{CONDITIONS CAUSING CHAOS IN AGC}

In this part the Melnikov method is used to argument the condition of the chaos in gauge control system

Equation (6) is equivalent to

$\left\{\begin{array}{c}\dot{x}=y \\ \dot{y}=-\mu y+\alpha x+\gamma x^{3}+\delta \cos \omega t\end{array}\right.$
Where, in the gauge control system, $\alpha>0, \gamma<0[5,6]$. This system has 3 singular points, the saddle point $F_{0}(0,0)$, stable focus $F_{1}(\sqrt{-\alpha / \gamma}, 0)$ and $F_{2}(-\sqrt{-\alpha / \gamma}, 0)$. If the system is not subject to the damping force and compulsion, that is $\mu=0$ and $\delta=0$, then system (7) is a Hamilton system, written as

$$
H(x, y)=\frac{1}{2} y^{2}-\frac{1}{2} \alpha x^{2}-\frac{1}{4} \gamma x^{4}=h
$$

Theorem 1 System (7) has sub-harmonic orbit when $\alpha>0$ and $\gamma<0$, and the parameter equation of the subharmonic orbit is

$$
x=\sqrt{\frac{-8 \alpha}{\gamma}} \frac{\sqrt{C} e^{\sqrt{\alpha / 2} t}}{1+C e^{\sqrt{\alpha} t}}
$$

Where, $C>0$ is a constant and is determined by the initial conditions

Proof From qualitative analysis, $F_{1}$ and $F_{2}$ are the center and $F_{0}$ is the saddle point, if $\alpha>0$ and $\gamma<0$, there is sub-harmonic trajectory passing by $(0,0)$. Taking $H=0$, from (8) gives 
$\frac{d x}{d t}=y= \pm \sqrt{\alpha x^{2}+\frac{1}{2} \gamma x^{4}}$

$\frac{d x}{\sqrt{\alpha x^{2}+\frac{1}{2} \gamma x^{4}}}= \pm d t$

Through integral: $\frac{1}{\sqrt{\alpha}} \ln \left|\frac{2 \sqrt{\alpha}-\sqrt{4 \alpha+2 \gamma x^{2}}}{2 \sqrt{\alpha}+\sqrt{4 \alpha+2 \gamma x^{2}}}\right|=t+C_{1}$,

Where, $C_{1}$ is determined by the initial conditions and $C_{1}=x(0)$

Then, $\frac{2 \sqrt{\alpha}-\sqrt{4 \alpha+2 \gamma x^{2}}}{2 \sqrt{\alpha}+\sqrt{4 \alpha+2 \gamma x^{2}}}=e^{\sqrt{\alpha}\left(C_{1}+t\right)}=C e^{\sqrt{\alpha} t}$.

The sub-harmonic orbit in the form of equation (9) is obtained. Proof is finished.

Lemma [7] Smale-Birkhoff sub-harmonic point theorem. $f: R^{n} \rightarrow R^{n}$ is a diffeomorphism, $p$ is its hyperbolic fixed point. If there exists a transversal intersection point $q \neq p$ of $W^{s}(P)$ and $W^{u}(P)$, then there is a hyperbolic invariant set in $f$ and $f$ is topologically equivalent to finite sub-shift mapping on $\Lambda$, that is chaos.

Introducing the notation, $a \wedge b=a_{1} b_{2}-a_{2} b_{1}$, and considering the Melnikov function

$$
\begin{aligned}
M\left(t_{0}\right)= & \int_{-\infty}^{+\infty}\left\{f\left(\bar{x}_{0}(t)\right) \wedge g\left(\bar{x}_{0}(t), t+t_{0}\right)\right. \\
& \left.\exp \left[-\int \text { trace }_{f} f\left(\bar{x}_{0}(s)\right) d s\right]\right\} d t
\end{aligned},
$$

Where, $\bar{x}_{0}(t)$ is the value of $x(t)$ for the case of nondamping, $D_{f} f\left(\bar{x}_{0}(s)=\left[\begin{array}{cc}\frac{\partial f_{1}}{\partial x_{1}} & \frac{\partial f_{1}}{\partial x_{2}} \\ \frac{\partial f_{2}}{\partial x_{1}} & \frac{\partial f_{2}}{\partial x_{2}}\end{array}\right]\right.$.

Let, $f_{1}=y, f_{2}=\alpha x+\gamma x^{3}, g_{1}=0, \quad$ and $g_{2}=\delta \cos \omega t-\mu y$, denote $\dot{x}=f(x)+\varepsilon g(x)$. By calculating, $\int$ trace $_{f} f\left(\bar{x}_{0}(t)\right)=0$, the Melnikov function can be written as $M\left(t_{0}\right)=\int_{-\infty}^{+\infty} f\left(\bar{x}_{0}(t)\right) \wedge g\left(\bar{x}_{0}(t), t+t_{0}\right) d t$.

According to the Smale-Birkhoff subharmonic point theorem, if there is a simple zero of $M\left(t_{0}\right)$ that does nothing to do with $\varepsilon$, there are some hyperbolic invariant sets of the iteration according to the poincare map and this map is chaotic in the meaning of Smale-horseshoe.
Theorem 2 The AGC system is chaotic in the sense of Smale-horseshoe if the parameters of the system satisfy the condition

$\frac{\delta}{\mu}>\frac{\sqrt{2}}{3 \pi \omega} \sqrt{-\frac{\alpha^{3}}{\gamma}} \cosh \frac{\pi \omega}{\sqrt{\alpha}}$

Proof According to the Melnikov function of the subharmonic orbit

$$
\begin{aligned}
& M\left(t_{0}\right) \quad=\int_{-\infty}^{+\infty} y(t)\left[\delta \cos \omega\left(t+t_{0}\right)-\mu y(t)\right] d t \\
& =-\mu \int_{-\infty}^{+\infty} y^{2} d t+\delta \int_{-\infty}^{+\infty} y \cos \omega\left(t+t_{0}\right) d t=-\mu I_{1}+\delta I_{2}
\end{aligned}
$$

Let's calculate ${ }^{I_{1}}$ and $I_{2}$ separately.

$$
\begin{aligned}
& I_{1}=\int_{-\infty}^{+\infty} y^{2} d t=\int_{-\sqrt{-2 \alpha / \gamma}}^{\sqrt{-2 \alpha / \gamma}} \dot{x} d x \\
& =\int_{-\sqrt{-2 \alpha / \gamma}}^{\sqrt{-2 \alpha / \gamma}} \sqrt{\alpha x^{2}+\frac{1}{2} \gamma x^{4}} d x=-\frac{4}{3 \gamma} \alpha^{\frac{3}{2}} \\
& I_{2}=\int_{-\infty}^{+\infty} y \cos \omega\left(t+t_{0}\right) d t \\
& =\sqrt{\frac{-8}{\gamma} \omega \int_{-\infty}^{+\infty} \frac{\sqrt{C} e^{-\sqrt{\alpha / 2} t}}{1+\sqrt{C} e^{-\sqrt{\alpha} t}} \sin \left[\omega\left(t+t_{0}\right)\right] d t}
\end{aligned}
$$

Denote that $f(z)=\frac{\sqrt{C} e^{-\sqrt{\alpha / 2} t}}{1+\sqrt{C} e^{-\sqrt{\alpha} t}} \sin \left[\omega\left(z+t_{0}\right)\right]$ and calculate the integral.

The singular point of function $f(z)$ is

$$
z_{k}=\frac{1}{\sqrt{\alpha}}\left(\ln \frac{1}{C}+i \pi\right) \quad k= \pm 1, \pm 3, \cdots
$$

And all the singular point is first-order point, selecting the integration path shown in Fig. (2). From the residue theorem

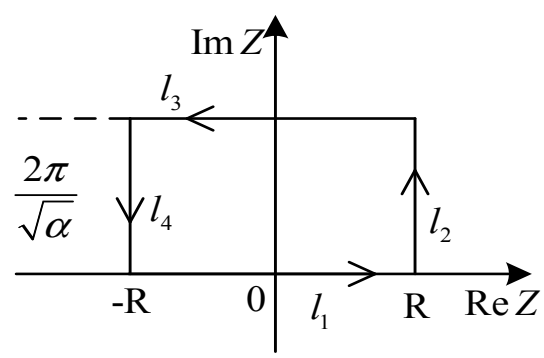

Fig. (2). Integaral path

$$
\oint f(z) d z=\int_{l_{1}} f(z) d z+\int_{l_{2}} f(z) d z+\int_{l_{3}} f(z) d z+\int_{l_{4}} f(z) d z
$$




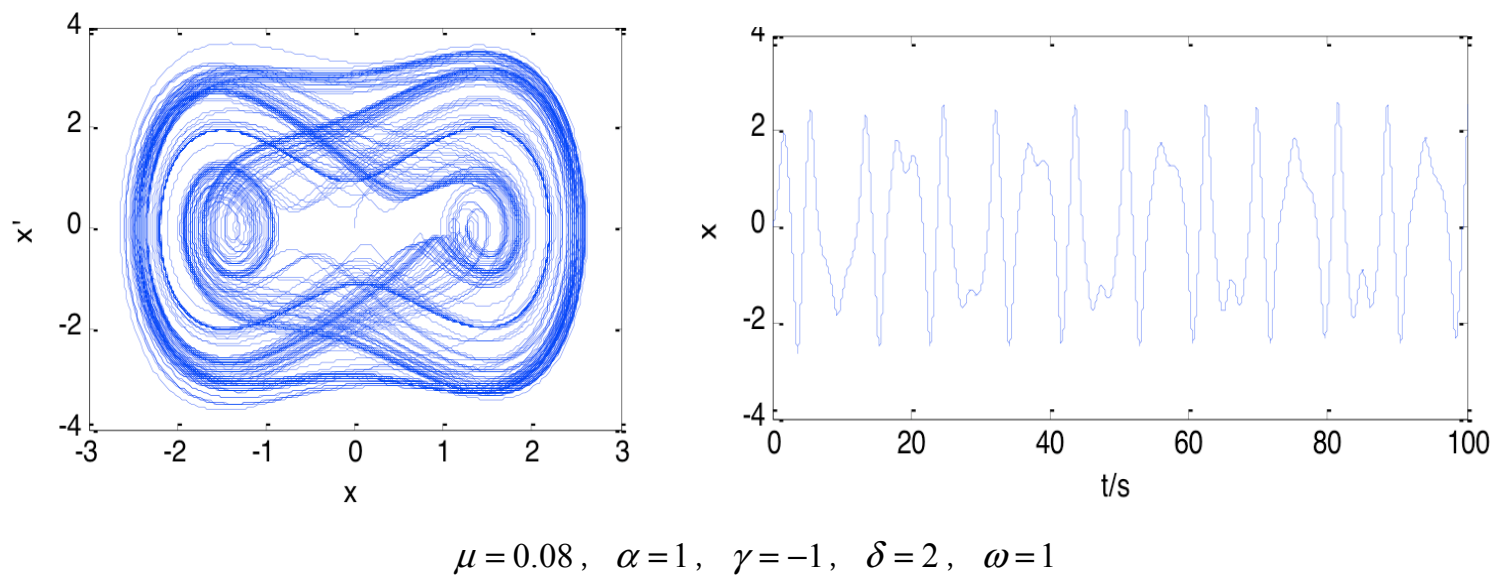

Fig. (3). Chaotic phase and time-domain graph with different parameters.

$$
\begin{aligned}
& \operatorname{Res}\left[f(z), z_{1}\right] \\
= & 2 \pi i \operatorname{Res}\left[f(z), z_{0}\right]=\lim _{z \rightarrow z_{0}}\left(z-z_{1}\right) \frac{\sqrt{C} e^{-\sqrt{\alpha / 2} z}}{1+\sqrt{C} e^{-\sqrt{\alpha} z}} \sin \left[\omega\left(z+t_{0}\right)\right] \\
= & -\frac{i}{\sqrt{\alpha}} \sin \left[\omega\left(z_{1}+t_{0}\right)\right] \\
= & -\frac{i}{\sqrt{\alpha}} \sin \omega\left(t_{0}+\frac{1}{\sqrt{\alpha}} \ln \frac{1}{C}\right) \cosh \frac{\pi \omega}{\sqrt{\alpha}}+ \\
& \frac{1}{\sqrt{\alpha}} \cos \omega\left(t_{0}+\frac{1}{\sqrt{\alpha}} \ln \frac{1}{C}\right) \sinh \frac{\pi \omega}{\sqrt{\alpha}} \\
& 2 \pi i \operatorname{Res}\left[f(z), z_{1}\right] \\
= & \frac{2 \pi}{\sqrt{\alpha}} \sin \omega\left(t_{0}+\frac{1}{\sqrt{\alpha}} \ln \frac{1}{C}\right) \cosh \frac{\pi \omega}{\sqrt{\alpha}}+ \\
& \frac{2 \pi}{\sqrt{\alpha}} i \cos \omega\left(t_{0}+\frac{1}{\sqrt{\alpha}} \ln \frac{1}{C}\right) \sinh \frac{\pi \omega}{\sqrt{\alpha}} \\
& \lim _{R \rightarrow+\infty} \int_{l_{1}} f(z) d z=\frac{1}{\omega} \sqrt{\frac{\gamma}{-8 \alpha}} I_{2} \\
\text { and } &
\end{aligned}
$$$$
\lim _{R \rightarrow+\infty} \int_{l_{2}} f(z) d z=\lim _{R \rightarrow+\infty} \int_{l_{4}} f(z) d z=0
$$$$
\begin{aligned}
\lim _{R \rightarrow+\infty} \int_{l_{2}} f(z) d z \mid & \leq\left|\int_{l_{2}} \frac{\sqrt{C} e^{-\sqrt{\alpha / 2} z}}{1+\sqrt{C} e^{-\sqrt{\alpha} z}} d z\right| \\
& \leq \int_{0}^{\frac{2 \pi}{\sqrt{\alpha}}} \frac{\sqrt{C} e^{-\sqrt{\alpha / 2} x_{1}}}{1+\sqrt{C} e^{-\sqrt{\alpha} x_{1}}} \\
& =\frac{2 \pi}{\sqrt{\alpha}} \frac{\sqrt{C} e^{-\sqrt{\alpha / 2} x_{1}}}{1+\sqrt{C} e^{-\sqrt{\alpha} x_{1}}}
\end{aligned}
$$$$
\lim _{R \rightarrow+\infty} \int_{l_{3}} f(z) d z
$$$$
=\int_{-\infty}^{+\infty} \frac{\sqrt{C} e^{-\sqrt{\alpha / 2} x}}{1+\sqrt{C} e^{-\sqrt{\alpha} x}} \sin \omega\left(x+t_{0}\right) \cosh \frac{2 \pi \omega}{\sqrt{\alpha}} d x+
$$$$
\int_{-\infty}^{+\infty} \frac{\sqrt{C} e^{-\sqrt{\alpha / 2} x}}{1+\sqrt{C} e^{-\sqrt{\alpha} x}} i \cos \omega\left(x+t_{0}\right) \sinh \frac{2 \pi \omega}{\sqrt{\alpha}} d x
$$$$
=\frac{1}{\omega} \sqrt{\frac{\gamma}{-8 \alpha}} \cosh \frac{2 \pi \omega}{\sqrt{\alpha}} I_{2}+i \sinh \frac{2 \pi \omega}{\sqrt{\alpha}} I_{3}
$$$$
\text { Where, } I_{3}=\int_{-\infty}^{+\infty} \frac{\sqrt{C} e^{-\sqrt{\alpha / 2} x}}{1+\sqrt{C} e^{-\sqrt{\alpha} x}} \cos \omega\left(x+t_{0}\right) d x
$$

Comparing the real part by the residue theorem

$$
I_{2}=\pi \omega \sqrt{\frac{-8}{\gamma}} \operatorname{sech} \frac{\pi \omega}{\sqrt{\alpha}} \sin \omega\left(t_{0}-\frac{\ln C}{\sqrt{\alpha}}\right)
$$

The Melnikov function is expressed as following

$$
M\left(t_{0}\right)=\mu \frac{4}{3 \gamma} \alpha^{\frac{3}{2}}+\delta \pi \omega \sqrt{\frac{-8}{\gamma}} \operatorname{sech} \frac{\pi \omega}{\sqrt{\alpha}} \sin \omega\left(t_{0}-\frac{\ln C}{\sqrt{\alpha}}\right)
$$

By the lemma, the condition that the system is chaotic in the sense of Smale-horseshoe is (10). Proof is finished.

Now in certain range of thickness spectrum widely used in code rolling mills of plate production, the simulation results with related parameters are shown as follows. The fundamental eccentricity frequency labels $\omega$, and $1 \leq \omega \leq 3$, As same time, $0.2 \leq \delta \leq 2,0.05 \leq \mu \leq 0.1$. When the condition satisfied with equation (10) and there are some simulations as shown in Fig. (3). From Fig. (3) it can be seen that $x$ is non-periodic although it is bounded, irregularly oscillating. When the system converts to one of the three motion 


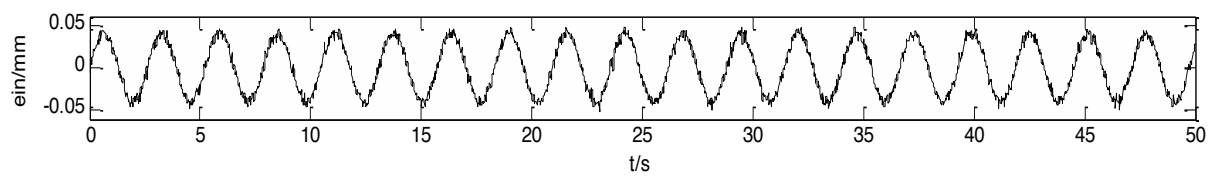

a.

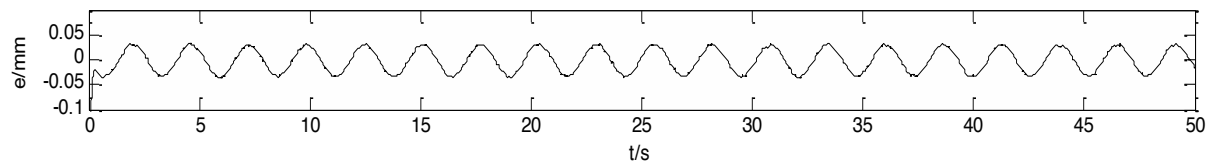

b.

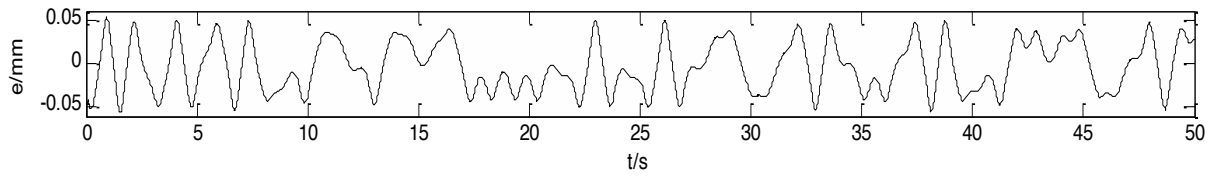

c.

Fig. (4). The deviation of gauge system caused by eccentricity

singularities surrounding a fixed point the periodic number shows stochastic behavior. The system will become chaotic when different periodic orbits are dense and any unstable periodic orbits meet.

\section{CHAOS CONTROL METHODS}

At the moment, in order to make up for the influence of roll eccentricity on thickness many methods have been used. These methods fall into three broad categories [8]. The first is passive and the main aim is to make roll gap system insensitive with the disturbance induced by the roll eccentricity. One of the classic techniques is dead-zone method by filtering the interference signal to prevent the adjustment misused. The second is active and in order to compensate the roll eccentricity it is used to determine the roll eccentricity and the roll gap controller. The spectrum analysis and identification method are popular. The third called prevent control method. It reduces the influence of roller eccentricity on thickness in rolling process usually. Three ways may be optimized when there isn't chaos caused by the eccentric disturbance in the gauge control system.

Considering the actual condition of AGC system, there is deviation about thickness caused by rough material bias and the eccentricity disturbance as shown in the diagram Fig. (4). As shown, in Fig. (4a) when the eccentricity is not controlled the deviation is smaller than $50 \mu \mathrm{m}$. Using the first method deviation of thickness is obviously reduced when there is not chaotic in the gauge control system. Knowing from Fig. (4b) The deviation can keep to less than $30 \mu m$. However, when the chaos caused by eccentricity exists in gauge control system, the thickness deviation will be more than $55 \mu \mathrm{m}$, and it does not reduce but increases as can be seen from Fig. (4c).

In the high precision rolling this is prohibited. So when the eccentricity disturbance brings the auto gauge control system into chaotic the rolling accuracy of the thickness will be under great perturbation. Without considering the impact of chaos the accuracy of thickness control cannot be achieved through the general methods. Through changing the rolling conditions chaos may be avoided. Changing the lubricating situation in the deformation zone to alter the damping coefficient, adjusting rhythm and speed of rolling to alter the eccentricity frequency, optimizing the parameters of the original controller and designing a new controller are all useful.

In order to test the effectiveness control methods, the parallel distributed compensation method (PDC) based on TS fuzzy model, the variable general universe fuzzy controller, and feedback linearization method (FL) are applied to restrain the chaotic in the AGC system. Using the parameters of the PDC as literature [9] and following the contractionexpansion factors of the VUFC $[10,11]$ the results of 4 kinds control methods are shown in Fig. (5). Other values of VUFC are equal to HACO-VUFC [12].

$$
\begin{aligned}
& \alpha_{1}(e)=\left(\frac{|e|}{E}\right)^{0.8}+0.001 \\
& \alpha_{2}(e c)=\left(\frac{|e c|}{E C}\right)^{0.9}+0.001
\end{aligned}
$$




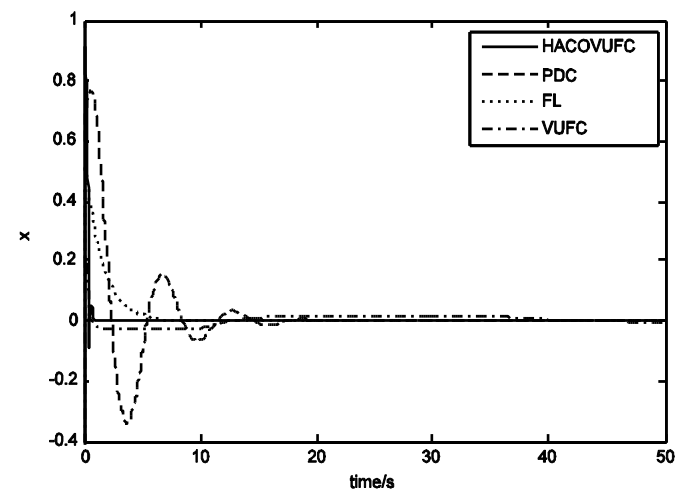

Fig. (5). System output of 3 control methods.

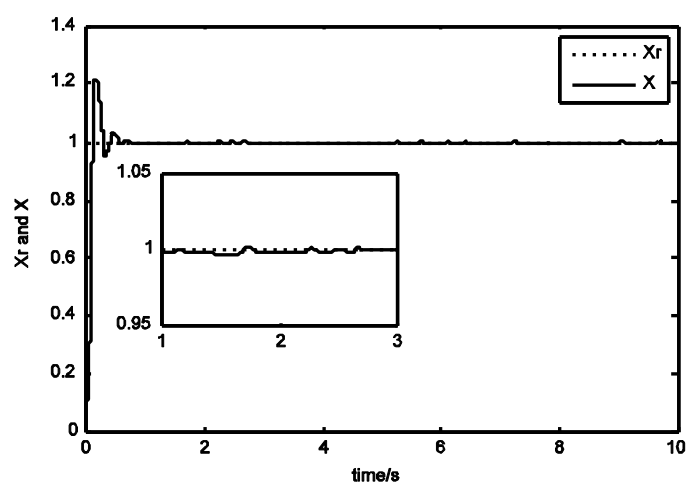

a

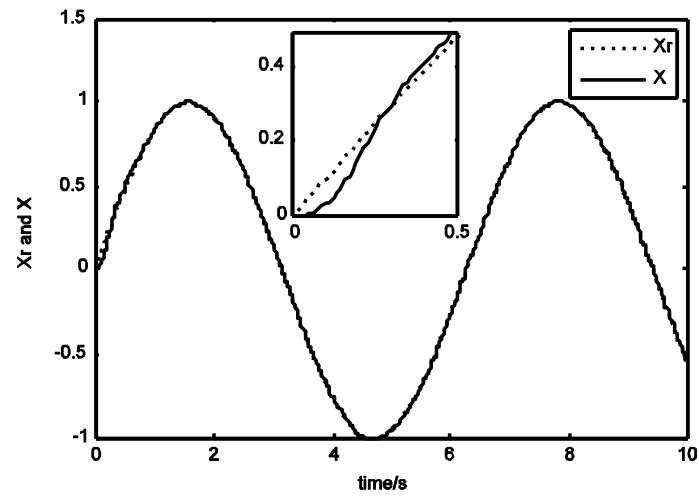

b

Fig. (6). Output of chaotic following control.

$\beta(e, e c)=\left(\frac{|e|}{E}\right)^{0.3}\left(\frac{|e c|}{E C}\right)^{0.9}$

In chaos control, literature $[13,14]$ proposed that besides the chaos stabilization and chaos synchronization, we should also consider the chaotic model following control (CMFC). The proposed method in this paper can also realize the CMFC and the simulation result is shown in Fig. (6). In Fig. (6), dotted lines are the given signal and solid line is the output of the chaotic system using HACO-VUFC method [15]. The step response of the system is shown in Figure 6a. It takes $0.4 \mathrm{~s}$ to make the system stable and in the local en- larged figure there is steady-state error within the allowable range of error. The result of the system tracking the sine curve is shown in Fig. (6b). From the local enlarged figure we can see that the system can track sinusoidal signal accurately in a very short period of time.

\section{CONCLUSION}

In this paper, it is proposed that the chaos phenomenon is possible because the disturbance of roll eccentricity has a stronger effect on thickness. As same time, chaotic conditions are proved based on the Melnikov function and 
Smale-horseshoe mapping in AGC system. Then several methods have been analyzed comparatively. By analyzing four kinds' contraction-expansion factors of variable fuzzy controller [16], it is suggested that HACO-VUFC method can overcome the contradiction between control accuracy and fuzzy control rules. Simulation shows that the product quality can be improved by chaos inhibition.

\section{CONFLICT OF INTEREST}

The authors confirm that this article content has no conflict of interest.

\section{ACKNOWLEDGEMENTS}

We wish to acknowledge the project about intelligent optimization algorithm system in the network security and law enforcement field of the people's public security university of china (2014JKF01137).

\section{REFERENCES}

[1] L. Zhang, K. Peng, and C. Tong, "Research on chaotic phenomenon in hot strip continuous rolling gauge control system", Control Engineering, no. 2, pp. 173-175, 2003.

[2] H.L. Shang, and J. Xu, "Delay induced multiple attractors and basins in controlled system", Dynamics of Continuous, Discrete \& Impulsive Systems B, vol. 14, no. Suppl 5, pp. 102-117, 2007.

[3] G. Chen, Controlling Chaos and Bifurcation in Engineering Systems," In: Boca Raton: CRC Press, pp. 46-65, 2000.
[4] V.B. Ginzberg, High-quality Steel Rolling, Beijing: Metallurgy Industry Press, 2000, pp. 95-118.

[5] S. Liu, and Y. Sun, "Identification and compensation of phase in roll eccentricity control," Metallurgy, no. 4, pp.42-43, 1994.

[6] W. Zhang, and J. Wang, "Argumentation of causing chaos in automatic gauge control systems," Journal of University of Science and Technology Beijing, vol. 33, no. 9, pp.1171-1176, 2011.

[7] Z. Liu, Analytic Method in Chaos Research, Shanghai: Shanghai University Press, 2000, pp. 95-124.

[8] Y. Li, X.H. Liu, J. Wang, X.L. Hu, K. Yan, and G.Y. Jin, “Analysis and prospect of roll eccentricity and its control", Steel rolling, no. 5, pp. 43-47, 2006.

[9] J. Qian, "Approach of chaotic control based on T-S fuzzy model", Journal of System Simulation, vol. 17, no. 12, pp. 2987-2990, 2005.

[10] H. Li, "Adaptive fuzzy controller based on variable universe", Science in China (Series E), vol. 29, no. 1, pp. 32-42, 1999.

[11] $\mathrm{H}$. Li, "A stable daptive fuzzy controller of nonlinear system based on variable universe", Science in China (Series E), vol. 32, no. 2, pp. 211-223, 2002 .

[12] H. Wang, W. Zhang, and A. Chang, "Chaos control algorithm via variable universe fuzzy controller in automatic gauge system", International Journal of Applied Mathematics and Statistics, vol.52, no. 2, pp. 228-235, 2014.

[13] K. Tanaka, "A unified approach to controlling chaos via an LMIbased fuzzy control system design", IEEE Transactions on Circuits and Systems, vol. 45, no. 10, pp. 1021-1040, 1998.

[14] K. Tanaka, "A unified approach to controlling chaos via an LMIbased fuzzy control system design", IEEE Transactions on Circuits and Systems, vol. 45, no. 10, pp. 1021-1040, 1998.

[15] M. Dorigo, and Gambardella L.M., "Ant colony system: a cooperative learning approach to the traveling salesman problem," IEEE Transactions on Evolutionary Computation, vol.1, no.1, pp. 53-66, Jan. 1997.

[16] W. Jing, and Z. Weiwei, "Chaos control via variable universe fuzzy theory in auto gauge control system," In: Proceedings of International Conference on Computer Application and System Modeling (ICCASM), vol. 1, 2010, pp. 363-369.

Received: November 19, 2014

Revised: December 11, 2014

Accepted: December 14, 2014

(C) Wang et al.; Licensee Bentham Open.

This is an open access article licensed under the terms of the Creative Commons Attribution Non-Commercial License (http://creativecommons.org/licenses/by-nc/3.0/) which permits unrestricted, non-commercial use, distribution and reproduction in any medium, provided the work is properly cited. 